\title{
Comparison and Realization of Fast Acquisition Method for GPS Signal under High Dynamic
}

\author{
Chan Zeng ${ }^{1}$, Weimin $\mathrm{Li}^{1}$, Bo $\mathrm{Bi}^{1}$ and Qiliang Chen ${ }^{1}$ \\ ${ }^{1}$ Dept. Navigation Chip, Beijing Microelectronics Technology Institute, Beijing, China
}

\begin{abstract}
In order to solve the problem of fast acquisition for GPS signal under high dynamic, the paper add window in traditional Partial Matched Filter (PMF) plus Fast Fourier Transform (FFT) for fast acquisition with the purpose of reducing the scalloping loss and improving the acquisition performance. And comparing the parallel code phase acquisition based on FFT with the new acquisition method based on the improved PMF+FFT. The high dynamic digital intermediate frequency signal is generated based on high dynamic trail. For the quicker acquisition speed, a method of fast blind search combining pseudo code correlation is used in acquisition. The results of simulation based on Matlab show that the new acquisition method can achieve the performance of $100 \mathrm{~g}$ dynamics.
\end{abstract}

\section{Introduction}

The change of Doppler frequency is very large if the time of acquisition is long under high dynamic, so the traditional receiver have been unable to meet the demand of high-speed run-time of navigation and positioning. The receiver has a very large velocity or acceleration and jerk which leading to a sharply change of Doppler and difficult to capture the GPS signal because of the bad environment under high dynamic. The traditional capture algorithms [1] such as sliding correlating which is two-dimension searching for the Doppler frequency and code phase, the method has large calculations and long search time so that it can't adapt to the high dynamic environment, the signal is easy to lose lock even if acquired, not to mention stably tracking. The receivers studied are in missile, rocket and satellite, therefore achieving fast and stable capture is the primary task of the study of the high dynamic receiver.

In order to achieve the acquisition of high dynamic, the goal is to capture as much as possible in shortest time, incorporating the speed of acquisition [2] and the resource consumption of hardware, the paper improves the structure of the PMF combining FFT of the laboratory. The PMF can effectively reduce the subsequent points of FFT, and FFT algorithm can compensate the problem of correlation peak steeply attenuated caused by the increase of Doppler frequency shift. So the PMF combining FFT not only greatly reduces the consumption of the hardware resource, but also speeds up the capture.
Finally, the paper compares the common acquisition method of parallel code phase based on FFT [3] with the proposed the improved method based on PMF+FFT. The performance comparison show that the new fast acquisition method can make the rapid and accurate capture for GPS signal under high dynamic.

\section{Digital intermediate frequency signal}

\subsection{Signal Model of GPS}

There are orthogonal modulated with $\mathrm{C} / \mathrm{A}$ code and $\mathrm{P}(\mathrm{Y})$ code on GPS L1 signal, it takes the form:

$$
\begin{aligned}
& s_{L 1}\left(t_{j}\right)=A \cdot C\left[t_{j}-\tau_{1}\left(t_{j}\right)\right] \cdot D\left[t_{j}-\tau_{1}\left(t_{j}\right)\right] \cdot \sin \left[2 \pi f_{L 1} t_{j}+\phi_{1}\left(t_{j}\right)\right]+ \\
& \frac{A}{\sqrt{2}} \cdot P\left[t_{j}-\tau_{1}\left(t_{j}\right)\right] \cdot W\left[t_{j}-\tau_{1}\left(t_{j}\right)\right] \cdot D\left[t_{j}-\tau_{1}\left(t_{j}\right)\right] \cdot \\
& \cos \left[2 \pi f_{L 1} t_{j}+\phi_{1}\left(t_{j}\right)\right]+n_{1}\left(t_{j}\right)
\end{aligned}
$$

Where $t_{j}$ is the time of sampling, $A$ is the amplitude of L1 C/A carrier, C is C/A PRN code, D is the $50 \mathrm{~Hz}$ navigation data bit stream, $\tau_{1}$ is the Pseudo Random Noise (PRN) code phase of L1, $f_{L 1}$ is the intermediate value of the $\mathrm{L} 1$ carrier frequency, $\phi_{1}$ is the carrier phase of $\mathrm{L} 1, \mathrm{P}$ is the known P PRN code, W is the encrypted W PRN code, $n_{1}$ is zero-mean discrete time Gaussian white noise of $\mathrm{L} 1$. 


\subsection{Simulating the Doppler Frequency of Carrier and Code}

The core problem of generating intermediate frequency is simulating the Doppler frequency shift of carrier and pseudo code under high dynamic. The Doppler frequency shift of satellite signal is caused by the relative motion between the connection of user and satellite receivers [4].

$$
f_{d}=f_{r}-f=f \cdot v \cdot(\cos \beta) / c
$$

Where $f_{r}$ is the reception frequency, $f$ is the transmitting frequency of satellite, $v$ is the velocity of the user receiver, $\beta$ is the angle between the direction of the receiver and the incoming signal, $c$ is the velocity of light, $f_{d}$ is the Doppler frequency.

The Doppler Effect has significant impact on carrier and pseudo code, the changes of Doppler are corresponding with the dynamic model reception moment in high dynamic environment. Assuming that the receiver has the acceleration $a$ and jerk $a^{\prime}$, the changes of Doppler can be expressed as following:

$$
\left\{\begin{array}{l}
f_{d}=f_{R F} \cdot v / c \\
f^{\prime}{ }_{d}=f_{R F} \cdot a / c \\
f^{\prime \prime}{ }_{d}=f_{R F} \cdot a^{\prime} / c
\end{array}\right.
$$

Where $f_{R F}$ is the RF frequency, $f_{d}^{\prime}$ is the change rate of the Doppler frequency, $f^{\prime \prime}$ is the second order change rate of the Doppler frequency.

So the computation formula of $f_{L 1}$ in (1) is as following:

$$
\begin{gathered}
f_{d}(t)=f_{\text {dinit }}+1 / 2 \cdot f_{d}^{\prime} t+1 / 6 \cdot f_{d}^{\prime \prime} t^{2} \\
f_{L 1}(t)=f_{I F 1}+f_{d}(t)
\end{gathered}
$$

Where $f_{\text {dinit }}$ is the initial value of the Doppler
In this paper, it is only the delay $\Delta d$ caused by the Doppler frequency shift to be considered, the delay is as follows:

$$
\Delta d=\frac{v(t) \cdot t}{c}=\frac{t}{c} \cdot\left(v+a t+1 / 2 a^{\prime} t^{2}\right)
$$

So the formula of the Doppler frequency shift for pseudo code is as follows.

$$
f_{c d}=f_{C} \cdot v / c=\frac{f_{d}}{f_{R F}} \cdot f_{C}
$$

Where $f_{C}$ is the nominal frequency of the pseudo code, $f_{c d}$ is the code frequency deviation of pseudo code. For the $\mathrm{C} / \mathrm{A}$ code of L1:

$$
f_{c d}=f_{d} / 1540
$$

It is obvious that the code frequency deviation of pseudo code should not be neglected under high dynamic.

The generation of pseudo code uses the code NCO to output the dynamic code rate. The code NCO is the phase accumulator composed by adder and register, which the working process is: first accumulation until overflow to zero, $\mathrm{M}$ is the phase control word, $f_{s}$ is the driven clock frequency, the register's count value of NCO increase M in each clock cycle, the value overflow to zero when it is maximum, it is a cycle, so the frequency of the cycle signal is:

$$
f_{o}=\frac{M \cdot f_{s}}{2^{N}}
$$

Where $\mathrm{N}$ is 32 to improve the frequency resolution, the formula (8) is the ode frequency deviation of pseudo code, so the frequency control word of PN code NCO:

$$
M=\frac{2^{N} \cdot\left(f_{C}+f_{c d}\right)}{f_{s}}
$$
frequency, $f_{I F 1}$ is the intermediate frequency of L1.

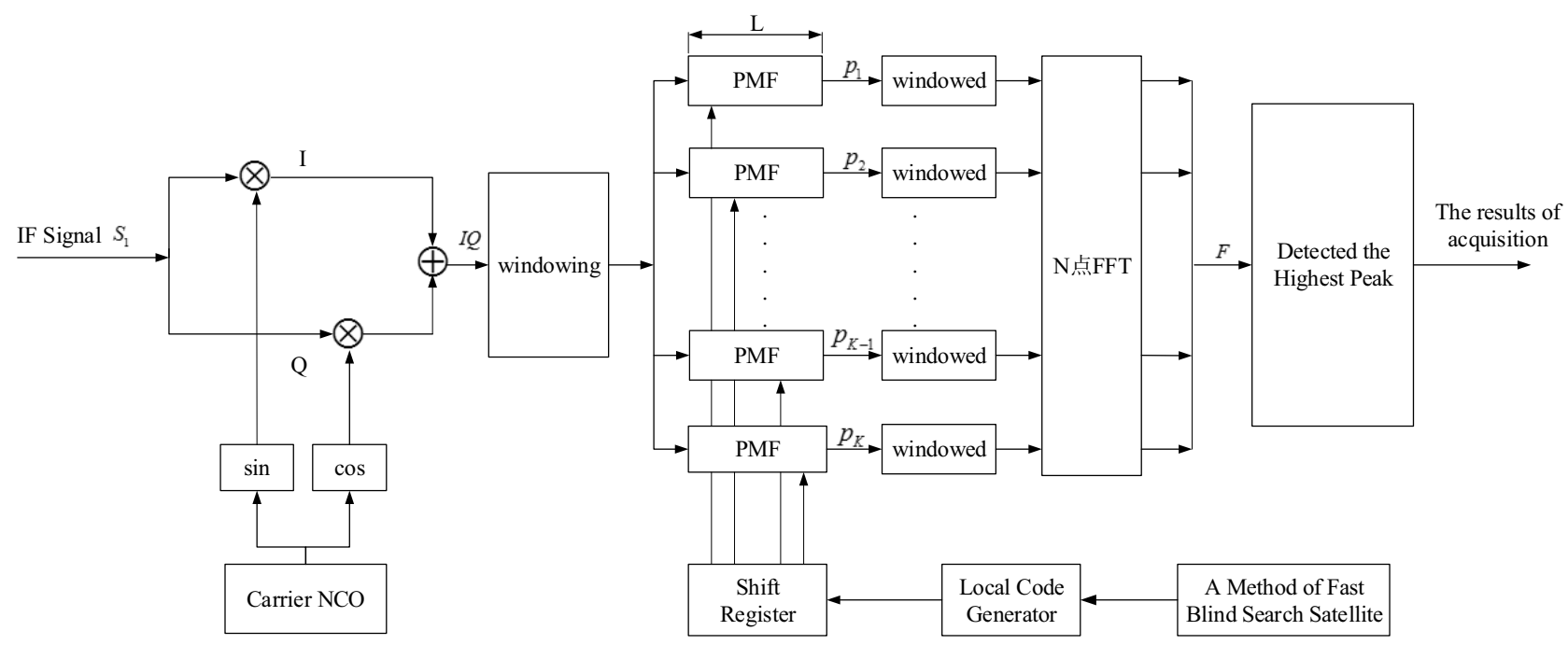

Figure 1. the structure of the improved PMF+FFT 


\section{Fast acquisition}

\subsection{The Improved PMF+FFT}

The acquisition method of PMF+FFT has been adopted in the laboratory, the PMF means to divide the coherent integration time $\mathrm{T}$ which decided by the frequency resolution into $\mathrm{K}$, it is done by the number of $\mathrm{K}$ PMF.As shown in Fig. 1 is the structure of the improved PMF+FFT, the intermediate frequency signal is demodulated by local carrier, and then by local pseudo-code, the range of Doppler frequency determine the $\mathrm{N}$ points of FFT. $M=T \cdot f_{C}$ is the total relevant calculations in the time of integration time $\mathrm{T}$, so the length of each matched filter is $L=T \cdot f_{C} / K$.

The code phase shifter will move code phase values to continue the above operations until all the code phase done. The correlative value of all code phase and frequency bands will be obtained, then use the adaptive threshold method to decide the correlation peak. It is through the principle of opening window to continue to detect the second peak when detected the highest peak. The ratio of the highest and the second peak is compared with threshold, if the ratio is greater, this satellite is visible. But the local code's phase is close to the received, so the peak detection need to set a window near the highest peak, the highest peak is as the center.

Assuming that the local pseudo-code is synchronized with received pseudo-code, the output of the n-th PMF is as follows:

$$
\begin{aligned}
& p(n)=\frac{1}{M} \sum_{k=(n-1) L+1}^{n L} e^{j\left(2 \pi \frac{f_{d}}{f_{C}} k+\varphi\right)} \\
& =\frac{1}{M} \frac{\sin \left(w_{d} L\right)}{\sin \left(w_{d}\right)} \cdot e^{j\left[2 w_{d}((n-1) L+1)+\varphi\right]}
\end{aligned}
$$

Where $w_{d}=\pi \cdot f_{d} / f_{C}$. The result of the number of $\mathrm{K}$ PMF are operated by $\mathrm{N}$ points FFT. It is available that the output normalized amplitude response of the FFT.

$$
\begin{aligned}
& \left|F\left(w_{d}, k\right)\right|=\left|\sum_{n=1}^{K} p(n) e^{-j \frac{2 \pi}{N} k n}\right|=\left|\frac{\sin \left(w_{d} L\right)}{\sin \left(w_{d}\right)}\right| \cdot\left|\sum_{n=1}^{K} e^{j\left(2 w_{d} L-2 \pi \frac{k}{N}\right) n}\right| \\
& =\frac{1}{M}\left|\frac{\sin \left(w_{d} L\right)}{\sin \left(w_{d}\right)} \cdot \frac{\sin \left(w_{d} M-\pi \frac{K}{N} k\right)}{\sin \left(w_{d} L-\pi \frac{k}{N}\right)}\right|
\end{aligned}
$$

The formula $F\left(w_{d}, k\right)$ can be divided into two parts:

$$
\begin{gathered}
F_{1}\left(w_{d}\right)=\frac{\sin \left(w_{d} L\right)}{L \cdot \sin \left(w_{d}\right)} \\
F_{2}\left(w_{d}, k\right)=\frac{\sin \left(w_{d} M-\pi \frac{K}{N} k\right)}{K \cdot \sin \left(w_{d} L-\pi \frac{k}{N}\right)}
\end{gathered}
$$

Where $F_{1}\left(w_{d}\right)$ is the influence of the amplitudefrequency response to the whole by PMF; $F_{2}\left(w_{d}, k\right)$ shows the FFT arithmetic contribution to the overall amplitudefrequency response.

Fig. 2 shows the normalized amplitude frequency response of the traditional PMF+FFT [5], as known from the figure, the FFT has played an important role in extension on the frequency for the amplitude-frequency response of PMF. The range of the Doppler frequency is expanded twice.

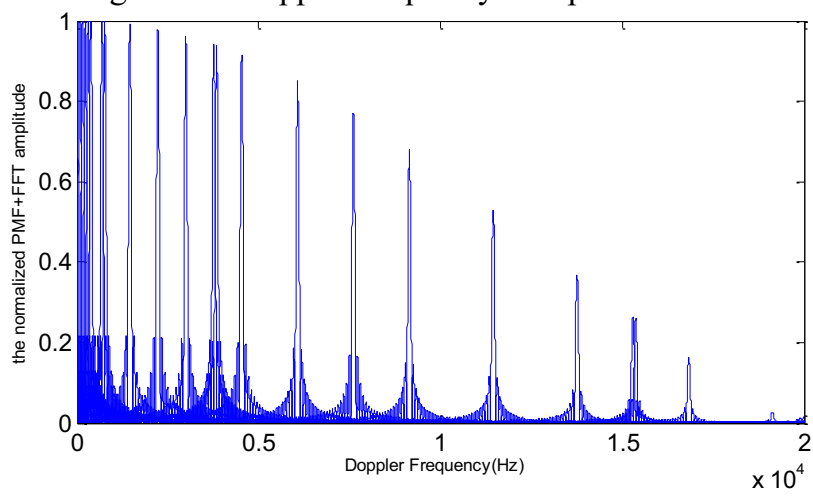

Figure 2. the normalized PMF+FFT amplitude frequency response

The shortcoming of PMF+FFT is the scallop loss [6] which makes the high false alarm rate and low performance of capture, so the improved is to reduce the scallop loss by using adding window. The window function can increase the main lobe width, thus to reduce the loss of scallops. For instance of hanning window:

$$
w(n)=\frac{1}{2}-\frac{1}{2} \cos \left(\frac{2 \pi n}{N}\right) \quad n=0,1,2, \ldots, N-
$$

The amplitude frequency response function of hanning window can be expressed as follows:

$$
W(w)=\frac{1}{2} W_{R}(w)+\frac{1}{4}\left[W_{R}\left(w-\frac{2 \pi}{N}\right)+W_{R}\left(w+\frac{2 \pi}{N}\right)\right]
$$

Where $W_{R}(w)$ is the amplitude characteristic of rectangular window which the length is $\mathrm{N}$.

Adding window on PMF can improve the low-pass characteristic. The same as hanning window, the parameters of system are multiplied by two to avoid amplitude attenuation. So the normalized frequency response of the input of PMF after adding window is:

$$
F_{1 w}\left(k, f_{d}\right)=F_{1}\left(f_{d}\right)+\frac{1}{2}\left[F_{1}\left(f_{d}-\frac{1}{L T_{c}}\right)+F_{1}\left(f_{d}+\frac{1}{L T_{c}}\right)\right]
$$

For the module of FFT, the introduction of window function is to reduce the scallop loss, the normalized frequency response of the output of FFT after adding window is:

$$
F_{2 w}\left(k, f_{d}\right)=F_{2}\left(k, f_{d}\right)+\frac{1}{2}\left[F_{2}\left(k, f_{d}-\frac{1}{M T_{c}}\right)+F_{2}\left(k, f_{d}+\frac{1}{M T_{c}}\right)\right]
$$

The formula (18) shows that the time-windowing of signal can be realized by linear operation in frequency domain.

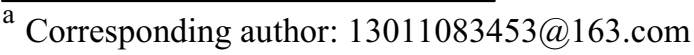


As shown in Fig. 3, it is the comparison the amplitude frequency response after adding window on PMF+FFT with no window. Considering the PMF and FFT after windowing, the simulation result shows that the improved algorithm not only reduce the scallop loss, but also improve the capture performance under high dynamic.

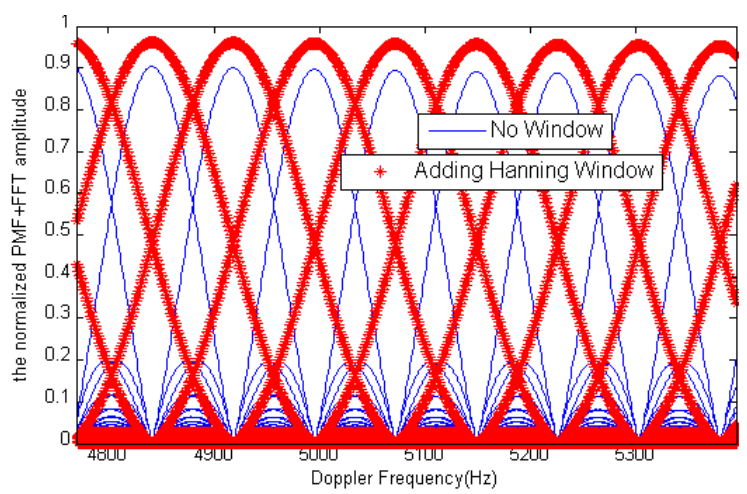

Figure 3. the normalized PMF+FFT amplitude frequency response after adding hanning window

\subsection{The Method of Fast Blind Searching for Satellite}

The receivers studied are in a state of blind search, there is no satellite ephemeris and almanac information to assist in search, the traditional search method is a waste of time that is searching 32 satellites in serial. Therefore, a method of fast blind search satellite combining pseudo code correlation is put forward. First, searching multiple satellites at the same time, if detected a correlation peak, then searching a single satellite from it, calculating the ratio between the highest peak and the second peak, and comparing this ratio with the threshold of acquisition to decide whether it is successful capture, until there are four satellites acquired. If searching four satellites at the same time, the search time will reduce $1 / 4$ at least for 32 satellites.

$$
C^{(\text {com })}(n)=\sum_{i=1}^{k} C^{(i)}(n)
$$

Where $C^{(i)}(n)$ is the pseudo code of satellite $i, C^{(\text {com })}(n)$ is the combination pseudo code of $\mathrm{k}$ satellites.

The pseudo-code generator copy the combination code of multiple satellites, then are correlated with received signal, which is equivalent to search and detect for the $\mathrm{k}$ satellites. If there is without a visible, the $\mathrm{k}$ satellites can be considered non-existent. Then the receiver can be turned to another $\mathrm{k}$ satellites, if one of more correlation peak, it can be decided that one or more satellite from the $\mathrm{k}$ satellites are visible, thus capturing in the $\mathrm{k}$ satellites until four satellites acquired.

\section{The parallel code phase acquisition}

\subsection{The Principle of Parallel Code Phase Acquisition}

The essence of the parallel fast capture [7] method based on FFT is that the convolution algorithms of time domain is converted into multiply arithmetic in frequency domain by Fourier transformation. It is that the two sequences $x(n)$ and $y(n)$ correlation arithmetic in the time domain are equivalent to their Fourier $X(k)$ and $\overline{Y(k)}$ multiplied in frequency domain. The method can calculate the all correlation value of code phases in each frequency band, equivalent to parallel correlator to improve the efficiency of the capture.

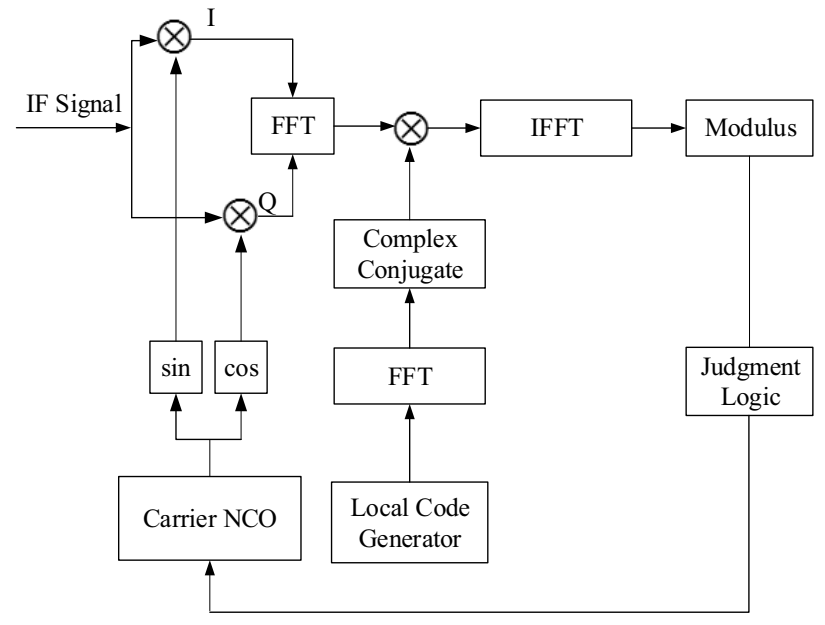

Figure 4. The parallel code phase search method based on FFT

As shown in Fig. 4, the digital intermediate frequency signal after mixing with local I and Q road carrier signal are combined into a plural form $I+j Q$ for FFT arithmetic. The transformation results are multiplied with the Fourier transform complex conjugate of local C/A pseudo-code, the product by IFFT operation can obtain the correlation result in time domain. Finally, detecting the peak of the correlation result after modulus, the peak compared with capture threshold method, if more than the capture threshold criterion captures the satellite signal. Then step the carrier frequency, continuing to the next band on the above process. The results by IFFT operation is all code phase correlation values on one frequency band after mixing with the local copied C/A pseudo code. In searching for the same satellite, its local copy C/A code of pseudocode phase is constant;it will transform the code phase of the local copy $\mathrm{C} / \mathrm{A}$ pseudo-code when searching different satellites.

\subsection{The Comparison of Two Methods of Acquisition}

Considering the acquisition in high dynamic environment, so the complexity time and space are used to 
evaluate the algorithm. The coherent integration time $T_{c o h}$, the range of doppler frequency $f_{\text {band }}$ is about $-20 \mathrm{kHz} \sim 20$ $\mathrm{kHz}$, and the sampling clock is $f_{s}$.

(a) Parallel Code Phase Acquisition based on FFT

If the $T_{c o h}=1 \mathrm{~ms}$, the number of sampling points are $N_{\text {sampl }}=f_{s} / 1000$. A search for a frequency and need two times FFT of $\mathrm{N}$ points and one time IFFT operations, so the computation is:

$$
N_{\text {sampl }}+3 N_{F F T}=\frac{f_{s}}{1000}+\frac{9 N}{2} \log _{2} N
$$

The total number of band are $N_{\text {freqbin }}$. If the search time of a band is the coherent integration time, the total time of searching all frequency bands is

$$
T_{\text {count }}=N_{\text {freqbin }} \times T_{\text {coh }}
$$

(b)The improved PMF+FFT

Assume that the number of PMF and FFT points are same as $\mathrm{N}$. The computation of searching for one code phase is:

$$
N_{\text {sampl2 }}+N_{F F T 2}=\frac{T_{c o h}}{N} \times f_{s}+\frac{3 N}{2} \log _{2} N
$$

For C/A the code chips are 1023, so if searched out all code phases the computation required is as following:

$$
N_{\text {count } 2}=1023 \times\left(T_{\text {coh }} \times f_{s}+\frac{3 N}{2} \log _{2} N\right)
$$

As all known, the all code phase of PMF is operated by pipelining in the coherent integration time, the operation of FFT can get the correlation value under all code phase and all frequency bands, so the time of PMF+FFT to complete a searching field is the coherent integration time:

$$
T_{\text {count } 2}=T_{\text {coh }}
$$

Through the above analysis of the computational cost and the time required, the parallel code phase acquisition based on FFT has small hardware resource consumption, but the points of FFT is very large and the time of search is long due to the serial searching for frequency. To the contrary, the PMF in the improved PMF+FFT achieve the purpose of the down sampling to greatly reduce the points of FFT, and adding window expand the range of frequency, the pipelining of PMF also accelerate the speed of acquisition.

To sum up, the improved acquisition method of PMF+FFT is the better choice to realize fast acquisition under the high dynamic.

\section{Experimental test results}

Assume the dynamism of the GPS receiver is that the initial velocity is $400 \mathrm{~m} / \mathrm{s}$ which corresponds to the initial Doppler frequency is $2100 \mathrm{~Hz}$, the acceleration is $100 \mathrm{~g}$ which corresponds to the rate of the Doppler frequency is $5146.372 \mathrm{~Hz} / \mathrm{s}, \mathrm{g}=9.8 \mathrm{~m} / \mathrm{s}^{2}$, the jerk is zero.
This paper simulates the improved algorithm PMF+FFT and the parallel code phase acquisition based FFT. Data about $10 \mathrm{~ms}$ was collected, theoretical value of the Doppler frequency is $2100+0.5 \cdot 5146.372 \cdot 0.01=2125.73 \mathrm{~Hz}$.

Fig. 5 and Fig. 6 show the acquisition reslut of improved PMF+FFT which has the obvious peak, the code phase offset and doppler frequency shift can be obtained by threshold decision. The code phase offse is correct and the doppler frequency shift is about $2075 \mathrm{~Hz}$, the error is within the frequency resolution about $76.85 \mathrm{~Hz}$.

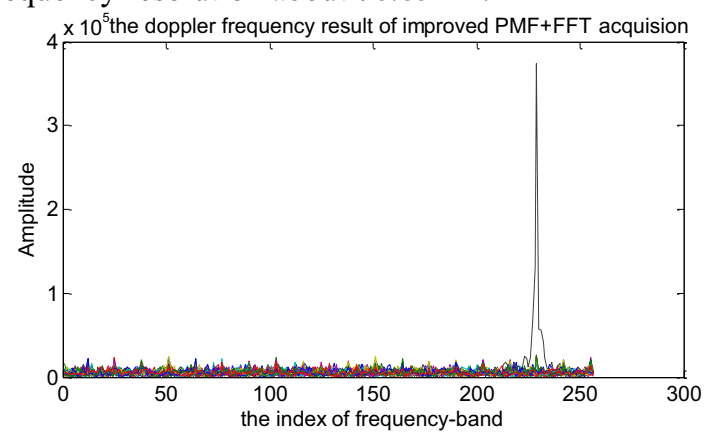

Figure 5. The Doppler frequency result of improved PMF+FFT acquisition

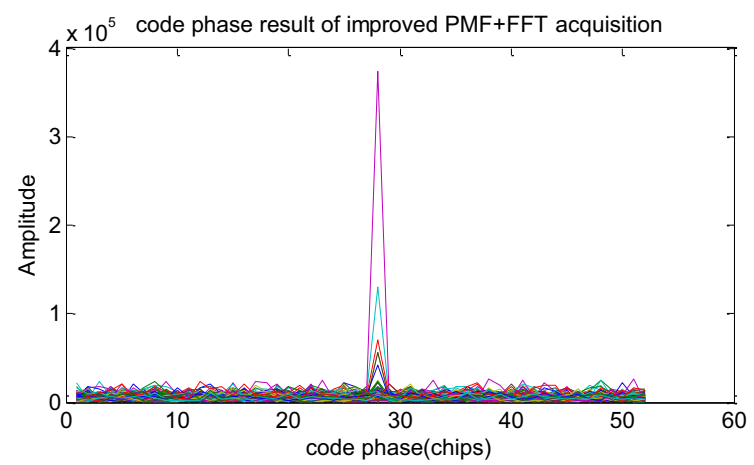

Figure 6. Figure of code phase result of improved PMF+FFT acquisition

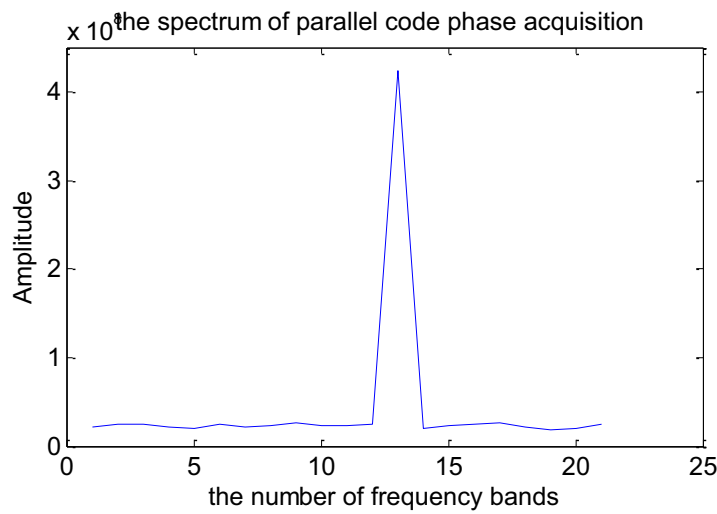

Figure 7. The Spectrum of parallel code phase acquisition

As shown in Fig. 7 ,it is the doppler spectrum figure after parallel code phase acquisition based on FFT. Set the the capture range of $20 \mathrm{KHz}$, and frequency step is $1000 \mathrm{~Hz}$, eventually the doppler frequency is $2000 \mathrm{~Hz}$, error about

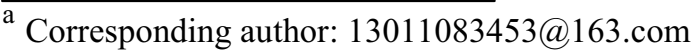


$126 \mathrm{~Hz}$, it is visible that acquisition accuracy of the parallel code phase trapping method based on FFT is not high.

\section{Summary and conclusions}

For the problem that rapidly and accurately acquisition GPS signal under high dynamic, there is a new method of high dynamic acquisition which is the fast acquisition based on improved PMF+FFT. The system simulation is tested in Matlab simulation platform using the source signals generated by simulator of the high dynamic signal. The simulator of the high dynamic signal can set dynamic movement of arbitrary acceleration and jerk. The improved PMF+FFT show that the scallops loss after adding window decrease by half, so the method can short acquisition time and improve the performance. Finally, the simulation results show that the proposed method is more accurate than the parallel code phase acquisition based on FFT. And it lays a good foundation for subsequent high dynamic tracking. The future work can fine spectrum on the basis of the results of the PMF+FFT, it will further improve the precision of acquisition.

\section{References}

[1] Zhou San-wen, Huang Long, Lu Man-hong. Acquisition of High Dynamic Spread Spectrum Signals with FFT [J]. Journal of Spacecraft TT\&C Technology, Vol24,P61-64 (2005).

[2] $\mathrm{Hu}$ Qiong. Research on the Acquisition Algorithm of High Dynamic and Weak GPS Signal [D].Shanghai: Shanghai Jiao Tong University (2013).

[3] Xie Gang. Principles of GPS and Receiver Design [M]. Beijing: Publishing House of Electronics Industry : P31 (2009)

[4] Bian C L,Ge H B,Chen R J.High-precision algorithm in PN code acquisition based on PMF-FFT[J]. Video engineering ,40(1):62-66 (2016).

[5] Nunes, F. D., Leitao J. M. N. A new fast code/frequency acquisition algorithm for GPS C/A signals[C]. Vehicular Technology Conference, 766-770 (2003).

[6] Zeng Qingxi,Tang Linlin,Wang Qing.Weak GPS L1 Signal Acquisition based on BPDC.[J].Transactions of Nanjing University of Aeronautics and Astronautics, Vol31,Jun, 3:331-337(2014).

[7] Li Yang, Xi XiaoLi. Combining FFT and Circular Convolution Method for High Dynamic GPS Signal Acquisition[J]. The Eighth International Conference on Electronic Measurement and Instruments, 159162(2007). 